\title{
TRANSPLANTE RENAL EM IDADE PEDIÁTRICA - 35 ANOS DE EXPERIÊNCIA DE UM CENTRO DE REFERÊNCIA
}

\section{Pediatric kidney transplantation - 35 years experience at a reference center}

\author{
Catarina Isabel Ribeiro', Rita Russo Belo², Joana Lorenzo ${ }^{3}$, Liane Correia Costa4, Ana Teixeira 4 , \\ Liliana Rocha ${ }^{4}$, Teresa Costa ${ }^{4}$, Paula Pires Matos ${ }^{4}$, Maria do Sameiro Faria ${ }^{4}$, Conceição Mota ${ }^{4}$
}

\section{RESUMO}

Introdução: Analisar a experiência em transplantação renal pediátrica do Centro Hospitalar Universitário do Porto (CHUP). Métodos: Estudo observacional retrospetivo single center, com inclusão dos doentes pediátricos submetidos a transplante renal entre janeiro de 1984 e novembro de 2019, na Unidade de Transplantação Renal do CHUP. Resultados: Efetuaramse 165 transplantes renais, no total de 157 doentes com idade inferior a 18 anos. Cento e cinquenta e um enxertos provieram de dador cadáver versus 14 de dador vivo. Realizaram-se 21 transplantes preemptive, estando a maioria dos doentes previamente sob técnica dialítica (50\% exclusivamente em diálise peritoneal). O tempo mediano de espera em técnica dialítica para transplante foi de 10 meses. Noventa e dois (59\%) doentes eram do género masculino, com idade mediana à data da intervenção de 13 anos (3 - 18). A principal etiologia da doença renal foi as anomalias congénitas do rim e do trato urinário (CAKUT) registadas em 58\%. Os dadores tinham idade mediana de 17 anos (1 - 58) e creatinina sérica terminal 1,00 mg/dL (0,30 - 2,10). A duração do internamento hospitalar foi 14 dias (4 - 60). Registaram-se 24 episódios de necrose tubular pós-transplante e 35 de rejeição aguda. A sobrevida do enxerto renal e do doente foi de $94 \%, 87 \%, 77 \%$ e $62 \%$ e de $99 \%, 98 \%, 98 \%$ e $93 \%$ aos 1, 5, 10 e 20 anos, respectivamente. A análise por subgrupos de décadas documentou taxas crescentes de sobrevida ( $p$-value <0.001). Conclusão: Os resultados obtidos corroboram a literatura e afirmam uma excelente sobrevida associada ao transplante renal pediátrico. Apesar de serem necessários estudos multicêntricos de maior impacto, esta análise retrata de forma holística a realidade nacional da modalidade.

Descritores: Transplante de Rim; Pediatria; Doença Renal Terminal; Análise de Sobrevida

Instituições:

1 Serviço de Nefrologia - Centro Hospitalar de Vila Nova de Gaia/ Espinho, Vila Nova de Gaia - Portugal

2 Serviço de Pediatria - Centro Hospitalar Trás-os-Montes e Alto Douro, Vila Real - Portugal

${ }^{3}$ Serviço de Pediatria - Centro Materno-Infantil do Norte - Centro Hospitalar Universitário do Porto, Porto - Portugal

${ }^{4}$ Serviço de Nefrologia Pediátrica - Centro Materno-Infantil do Norte - Centro Hospitalar Universitário do Porto, Porto - Portugal

\section{Correspondência:}

Catarina Isabel Correia Dias Ribeiro

Centro Hospitalar de Vila Nova de Gaia/Espinho - Unid.I - R. Conceição Fernandes, s/n - 4434-502, Vila Nova de Gaia, Porto - Portugal +351910771837

catarina.isabel.ribeiro@gmail.com

Recebido em: 28/11/19

Aceito em: 13/01/2020

\section{INTRODUÇÃO}

$\mathrm{Na}$ doença renal crónica, a perda progressiva da função renal conduz à inevitável necessidade de técnica substitutiva, constituindo o transplante renal a terapêutica de eleição. O transplante renal em idade pediátrica, realizado pela primeira vez com sucesso na década de 50 , é a modalidade com melhor relação custo-efetividade, capaz de conferir aos doentes portadores de doença renal avançada maior qualidade de vida e sobrevida., ${ }^{1,2}$

Contudo, o doente pediátrico possui particularidades singulares, que tornam a transplantação renal, nesta faixa etária, um maior desafio. Quando comparado com a idade adulta, a imaturidade do sistema imunológico predispõe por si só a um risco acrescido de processos infeciosos e linfoproliferativos, passíveis de se associarem a outras complicações, nomeadamente do foro hemodinâmico e vascular. ${ }^{3}$ Atualmente, admite- 
se que a seleção adequada de dadores, a preparação antecipada do procedimento cirúrgico e o aumento da experiência da equipa em muito potenciam um melhor resultado final. A acrescer a isso, a utilização de terapêuticas imunossupressoras mais potentes e o melhor conhecimento da sua ação farmacocinética contribuem também para uma melhor sobrevida do enxerto renal e do seu recetor. ${ }^{4}$ Ainda a sobreporse, reconhece-se que o sucesso do transplante renal pediátrico está condicionado por inúmeros outros fatores de ordem psicossocial, inerentes intrinsecamente ao próprio doente, e com impacto no desfecho final. ${ }^{5}$

De acordo com o registo português de transplantação renal, o CHUP é, até ao presente, a Unidade nacional com maior número de transplantes renais realizados em recetores com menos de 18 anos de idade. ${ }^{6,7} \mathrm{Com}$ o presente trabalho, os autores pretendem analisar a experiência do programa de transplantação renal pediátrica do CHUP ocorrida desde o início da sua atividade, e avaliar as características e perfil evolutivo do mesmo.

\section{MÉTODOS}

Os autores apresentam um estudo observacional retrospetivo single center, que incluiu a análise dos doentes pediátricos, com idade inferior a 18 anos à data da intervenção, submetidos a transplante renal entre 1 de janeiro de 1984 e 30 de novembro de 2019 na Unidade de Transplantação Renal do CHUP. Para isso, foi realizada a colheita de dados por consulta dos seus processos através do SClínico ${ }^{\circledR}$ e Sistema de Apoio ao Médico, procedendo-se posteriormente à análise descritiva dos dados epidemiológicos (incluindo número total de transplantes, género e idade do recetor e do dador à data do transplante) e clínicos (incluindo etiologia da doença renal crónica, serologias, tipo de dador, compatibilidades, tempo de isquemia, imunossupressão utilizada, duração de internamento, evolução e sobrevida).

A análise estatística foi efetuada através do software informático IBM SPSS Statistics ${ }^{\circledR}$ V24. As variáveis contínuas foram descritas com apresentação da mediana (percentil 25, P25 e percentil 75, P75). As curvas de sobrevida foram efetuadas com base na estimativa de Kaplan-Meier e a comparação de subgrupos através do Log-rank Test.

\section{RESULTADOS}

O início da atividade de transplantação renal pediátrica no CHUP ocorreu em janeiro de 1984, tendo sido efetuados até novembro de 2019, 165 transplantes em
157 doentes. Em 151 (91\%) casos o enxerto renal foi proveniente de um dador cadáver, contrastando com 14 (9\%) transplantes de doação em vida. Vinte e um doentes realizaram transplante renal preemptive. A maioria dos doentes $(n=136,87 \%)$ foi submetida a diálise prévia ao transplante, com um tempo mediano de 10 meses (P25 - P75, 6 - 20) em técnica dialítica. Dos doentes que realizaram tratamento dialítico prévio, $50 \%(n=68)$ realizaram exclusivamente diálise peritoneal, 33\% $(n=$ $45)$ exclusivamente hemodiálise e os restantes $(n=23)$ ambas as modalidades.

Relativamente à causa principal da doença renal, as CAKUT constituíram a etiologia mais frequente, em 95 $(58 \%)$ casos, seguidas das glomerulopatias (Tabela 1 ).

Tabela 1: Etiologia da doença renal

\begin{tabular}{lc}
\hline \multicolumn{1}{c}{ Variáveis, $\mathrm{n}$} & $(\mathrm{N}=165)$ \\
\hline CAKUT & 95 \\
Glomerulopatias (incluindo GESF) & $33(17)$ \\
Ciliopatias & 10 \\
Tubulopatia/ doença tubulo-intersticial & 7 \\
Síndrome hemolítico-urémico & 2 \\
Indeterminada & 10 \\
Outras & 8 \\
\hline
\end{tabular}

Legenda: CAKUT: anomalias congénitas do rim e do trato urinário; GESF: glomerulosclerose segmentar e focal

A idade mediana dos doentes à data da intervenção foi de 13 anos (3 - 18), com 92 (59\%) destes do género masculino. No que respeita aos dadores, a mediana de idades encontrada foi 17 anos (1 - 58), com a maioria $(n=116,70 \%)$ também do género masculino.

Todos os doentes submetidos a transplante apresentavam compatibilidade ABO. A maioria dos recetores $(n=99$, $60 \%$ ) era do grupo sanguíneo $A$, seguido do grupo $O$ em $33 \%(n=55)$. À data de transplantação, todos os doentes tinham marcadores de hepatite e vírus da imunodeficiência humana negativos, excetuando-se 2 com positividade para a hepatite B e 2 para a hepatite C. Treze por cento dos doentes portadores de pesquisa negativa para citomegalovírus apresentavam dador com positividade serológica para o mesmo agente (Tabela2).

Tabela 2: Pares serológicos de citomegalovírus

\begin{tabular}{c|c}
\hline Par Dador/Recetor & $\%$ \\
\hline D (-)/R (-) & 10 \\
D (+) / R (-) & 13 \\
D (-)/R(+) & 27 \\
D (+) / R (+) & 50 \\
\hline
\end{tabular}

Legenda: D: dador; $R$ : recetor; -: negativo; +: positivo 
Catarina Isabel Ribeiro, Rita Russo Belo, Joana Lorenzo, Liane Correia Costa, Ana Teixeira, Liliana Rocha, Teresa Costa, Paula Pires Matos, Maria do Sameiro Faria, Conceição Mota

À data do transplante renal, o valor mediano de ureia e creatinina sérica terminal do dador era de $45 \mathrm{mg} / \mathrm{dL}(10$ - 130) e 1,00 mg/dL (0,30 - 2,10), respetivamente.

A técnica cirúrgica utilizada consistiu na abordagem extraperitoneal, com incisão oblíqua no hipogastro; nos doentes mais pequenos (inferior a $15 \mathrm{Kg}$ ) a opção foi a incisão de Rutherford-Morrison. O tempo mediano de isquemia fria foi 23 horas (3 - 32).

No que respeita à terapêutica imunossupressora de indução, entre 1984 e 1989 a Metilprednisolona / Prednisolona, a Azatioprina e a Ciclosporina foram os fármacos mais amplamente utilizados. Em 1990 foi introduzido no protocolo da Unidade a globulina anti-timócito e os anticorpos monoclonais anti-IL2R (CD25), como o Daclizumab, e mais tarde o Basiliximab. Em 2002 o Micofenolato de Mofetil veio substituir a Azatioprina e dois anos depois passou-se a incluir outro inibidor da calcineurina, o Tacrolimus, como alternativa à Ciclosporina. A imunossupressão de indução mais realizada foi com corticoide, globulina anti-timócito, Micofenolato de Mofetil e Tacrolimus ( $n=55,33 \%$ ). Os resultados globais encontram-se expressos na Tabela 3.

Tabela 3: Esquema de Imunossupressão de indução

\begin{tabular}{l|c}
\hline \multicolumn{1}{c|}{ Variáveis, $\mathrm{n}$} & (N=165) \\
\hline Corticoide + AZA + CyA & 28 \\
Corticoide + ATG + CyA & 52 \\
Corticoide + ATG + AZA + CyA & 11 \\
Corticoide + ATG + MMF + CyA & 5 \\
Corticoide + ATG + MMF + Tacrolimus & 55 \\
Corticoide + Daclizumab + MMF + Tacrolimus & 3 \\
Corticoide + Basiliximab + MMF + Tacrolimus & 11 \\
\hline
\end{tabular}

Legenda: AZA: Azatioprina; CyA: Ciclosporina; ATG: globulina antitimócito; MMF: Micofenolato de Mofetil

Durante o período de internamento, verificou-se necessidade de suporte transfusional de hemoderivados, com unidades de concentrado eritrocitário, em 57 (35\%) casos, 23 dos quais no tempo intra-operatório.

No seguimento destes doentes durante o tempo hospitalar, registaram-se 24 casos de necrose tubular aguda, com necessidade transitória de hemodiálise, com uma mediana de 4 sessões (1 - 11). Obtiveramse 35 situações de rejeição aguda, com tempo de surgimento de 18 dias (6-180) após o transplante. A duração mediana de permanência hospitalar em internamento foi 14 dias (4 - 60).

No final do primeiro ano a sobrevida do enxerto renal e do doente foi $94 \%$ e $99 \%$, respetivamente. Aos 5, 10 e 20 anos a sobrevida foi $87 \%, 77 \%$ e $62 \%$ para o enxerto renal, e $98 \%, 98 \%$ e $93 \%$ para o doente (Figuras 1 e 2 ).
Figura 1: Sobrevida do enxerto renal, com curva de Kaplan-Meier

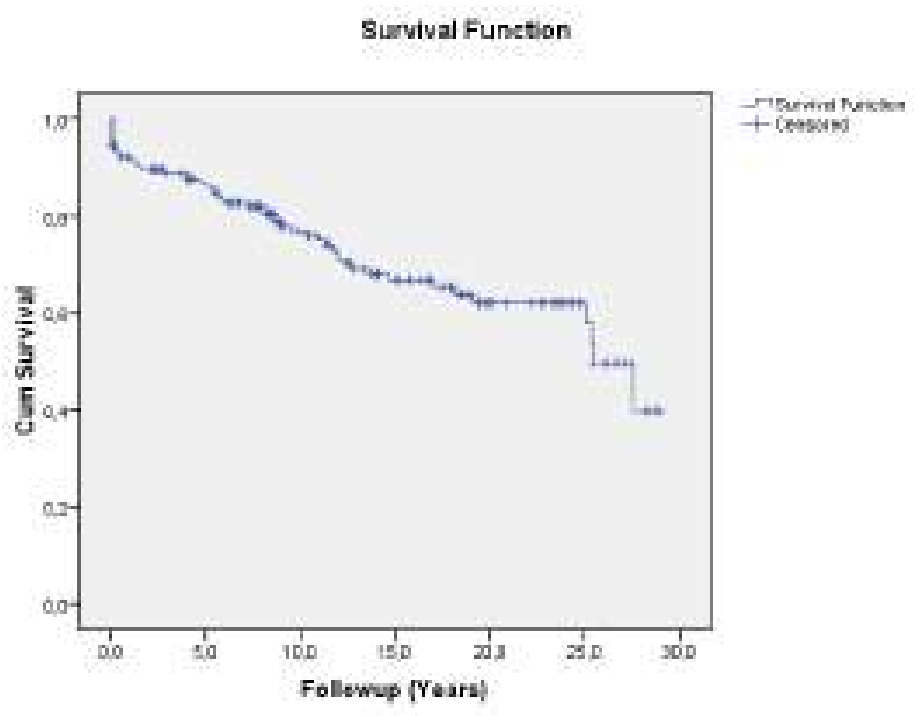

Figura 2: Sobrevida do recetor, com curva de Kaplan-Meier Surviral Function

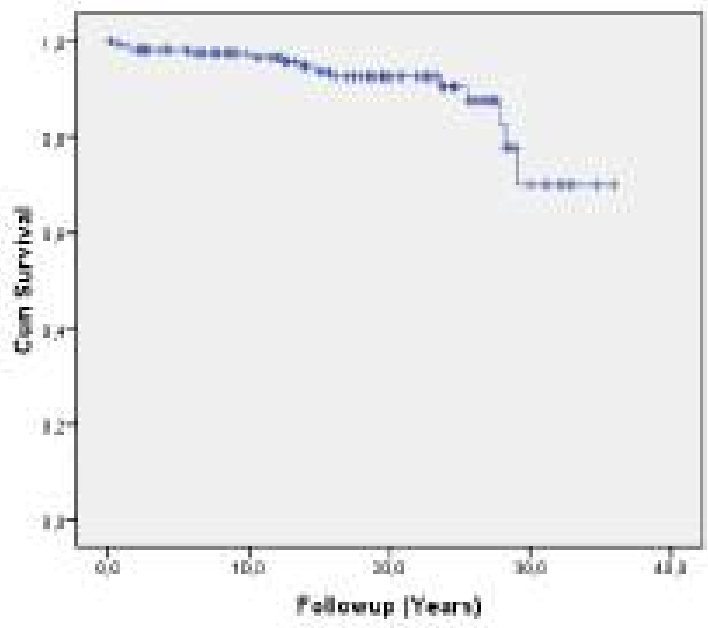

Touveriventest T Cerased

Numa tentativa de avaliar os resultados consoante um perfil temporal cronológico, foram considerados 4 períodos: 1984-89, 1990-99; 2000-09; 2010-19. No período compreendido entre 1984 e 1989, e nas décadas de 1990-99, 2000-09 e 2010-19 realizaram-se respetivamente, 13, 53, 52 e 47 transplantes renais. Em 146 doentes foi o primeiro transplante, em 10 doentes foi o segundo e em 1 doente, o terceiro.

Considerando estes 4 períodos efetuou-se a análise da sobrevida por subgrupos. Compararam-se as diferentes curvas de sobrevida através do Log-rank Test, constatando-se taxas crescentes ao longo do tempo, com aquisição de resultados estatisticamente significativos ( $p$-value <0.001) (Figura 3). 
Figura 3: Sobrevida do recetor por intervalos de tempo, com curva de Kaplan-Meier

Survival Functions

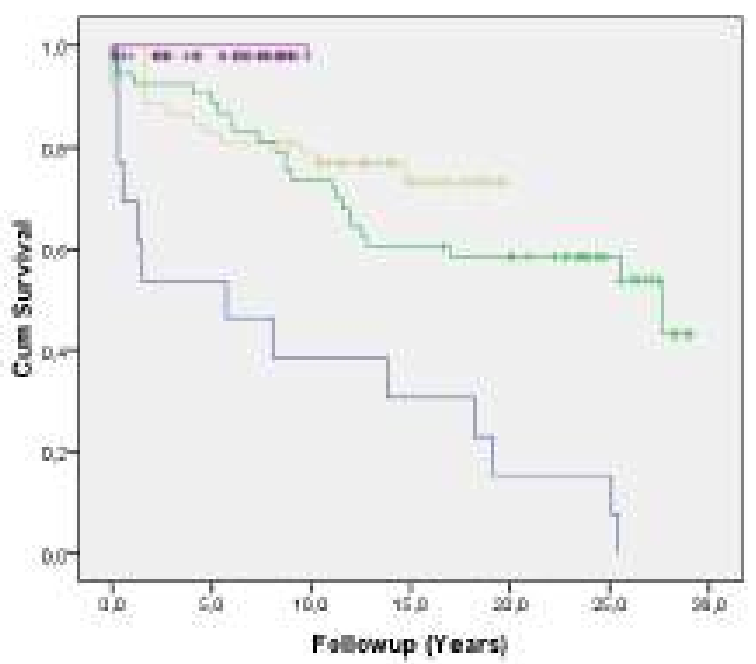

DECADAS

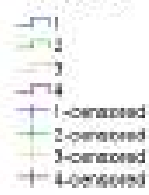

Legenda: Décadas 1: 1984-1989: 2: 1990-1999; 3: 2000-2009;4: 2010-2019)

A nefropatia crónica do enxerto correspondeu à maior causa de falência do transplante renal, em $69 \%$ dos casos. No total de doentes transplantados, registaramse $12(7,6 \%)$ óbitos, com idade mediana 26 (6 - 44) anos. Três doentes faleceram em idade pediátrica, com 6,12 e 14 anos, nenhum com enxerto renal funcionante, todos em programa crónico de hemodiálise. A sépsis, a doença cardiovascular e as neoplasias (adenocarcinoma do pulmão e linfoma B difuso de grandes células) estiveram entre as principais causas conhecidas (Tabela 4).

Tabela 4: Causas de óbito do recetor

\begin{tabular}{lcc}
\hline \multicolumn{1}{c}{ Etiologia } & $(\mathbf{N = 1 2 )}$ & $\begin{array}{c}\text { Idade do óbito } \\
\text { (anos) }\end{array}$ \\
\hline Sépsis & 3 & $12 ; 24 ; 27$ \\
Doença cardiovascular & 2 & $6 ; 44$ \\
Neoplasia & 2 & $28 ; 39$ \\
Acidente vascular cerebral & 1 & 21 \\
Hipercalémia (não compliance) & 1 & 14 \\
Peritonite esclerosante & 1 & 36 \\
Desconhecida & 2 & $26 ; 37$ \\
\hline
\end{tabular}

Findo o período de follow-up, 131 (83,5\%) doentes mantinham seguimento em consulta hospitalar de Nefrologia - Transplante Renal, 13 (8,2\%) doentes integraram programa crónico de hemodiálise e 1 de diálise peritoneal. Dos doentes que mantiveram seguimento no CHUP, o valor mediano de creatinina sérica à data da última consulta era $1,37 \mathrm{mg} / \mathrm{dL}(0,43$ $-5,70)$.

\section{DISCUSSÃO}

Nestes 35 anos em análise, realizaram-se nesta Unidade de Transplantação Renal 165 transplantes renais pediátricos, número este considerável face às estatísticas de transplantação pediátrica mundial. ${ }^{8}$

A mediana de idades dos recetores à data da intervenção (13 anos) reflete a realidade nacional, dado este tipo de transplante ser pouco frequente na primeira meia década de vida. Tradicionalmente e dadas as dificuldades técnicas inerentes ao próprio ato cirúrgico per si, o transplante renal em doentes com peso inferior a $10 \mathrm{Kg}$ não está recomendado, realizando-se apenas em situações excecionais.

A etiologia da doença renal varia consoante o grupo etário, diferindo notoriamente da população adulta na qual a diabetes mellitus se sobressai ocupando o lugar cimeiro. Segundo o North American Pediatric Renal Trials and Collaborative Studies, as etiologias que mais frequentemente motivam a terapêutica substitutiva da função renal são a agenesia, hipoplasia e displasia renal, seguidas da uropatia obstrutiva e da glomerulosclerose focal segmentar. ${ }^{9} \mathrm{Na}$ presente análise não foi diferente, com as CAKUT e as doenças glomerulares a destacaremse entre as mais comuns.

Embora não constante nos principais objetivos do trabalho, os autores admitem o pequeno número de transplantes preemptive como fator limitativo numa possível comparação do perfil evolutivo entre doentes com e sem diálise prévia ao transplante. No presente trabalho, a maioria dos doentes $(n=136,87 \%)$ foi submetida a diálise prévia ao transplante (com um tempo mediano de 10 meses em técnica dialítica), o que poderá predizer à posteriori um maior risco de complicações, nomeadamente do foro imunológico e cardiovascular. Estudos de maiores dimensões apresentam a transplantação renal preemptive como um preditor de melhor prognóstico e outcome final a médio e longo prazo. ${ }^{10}$

O baixo número de dadores vivos $(n=14)$ é ainda outro aspeto a citar. A transplantação renal de dador vivo apresenta-se com inequívocas vantagens, inclusive ao nível da função dos enxertos a médio e longo prazo. ${ }^{11}$ Também aqui o pequeno número de doentes com dador vivo presente nesta série não permitiu estabelecer ou extrapolar conclusões. Os autores consideram que o combate à lista de espera poderá ser notoriamente conseguido fomentando e difundindo protocolos de atuação de doação em vida nesta faixa etária e capacitando profissionais treinados para a sua colocação em prática.

A nefropatia crónica do enxerto correspondeu à maior causa de falência do transplante renal, o que está 
Catarina Isabel Ribeiro, Rita Russo Belo, Joana Lorenzo, Liane Correia Costa, Ana Teixeira, Liliana Rocha, Teresa Costa, Paula Pires Matos, Maria do Sameiro Faria, Conceição Mota

também de acordo com dados descritos na literatura.4,5 Findo o tempo de follow-up, os autores obtiveram taxas de sobrevida (do doente e enxerto renal) superiores à média registada em outras séries publicadas. Estes dados poderão ser resultado dos métodos de atuação da Unidade, extensamente protocolados e revistos, associados ao advento de avanços tecnológicos e à experiência cumulativa da equipa.

Os resultados obtidos corroboram mais uma vez a literatura, afirmando o transplante renal capaz de conferir excelentes taxas de sobrevida do enxerto renal e do seu recetor. A análise de sobrevida por subgrupos, veio demonstrar uma sobrevida crescente ao longo das últimas décadas ( $p$-value $<0.001$ ), o que está de acordo com uma melhoria também esta crescente dos cuidados prestados, a par da inovação farmacológica e do constante aperfeiçoamento de técnicas.

Em idade pediátrica, a doença renal crónica classificada em grau 5 pela Kidney Disease Improving Global Outcomes associa-se a um risco de mortalidade cerca 30 vezes superior ao da população geral na mesma faixa etária. ${ }^{1,2} \mathrm{Na}$ presente análise, apesar de se terem registado apenas 3 óbitos em idade pediátrica, verificouse uma mortalidade significativa $(7,6 \%)$ em idade jovem, 26 anos (6 - 44). Este facto leva os autores a relembrarem e reforçarem o peso que a doença renal acarreta no prognóstico vital do grupo de doentes que dela sofre.

\section{CONCLUSÃO}

Apesar do caráter retrospetivo e single center do presente trabalho, reconhece-se a importância de uma possível inclusão destes doentes em registos internacionais, que permitam estudos futuros de maior impacto. Apesar de algumas limitações, dado o importante número de doentes pediátricos submetidos a transplante renal nesta Unidade, e sendo o CHUP, até ao presente, a Unidade com mais transplantes realizados em Portugal, os autores admitem a presente análise capaz de ecoar e refletir de forma holística, a realidade nacional desta modalidade.

\section{ABSTRACT}

Introduction: To analyze the experience at Oporto University Hospital Center (CHUP) in pediatric kidney transplantation. Methods: Retrospective single center observational study, including pediatric kidney transplant patients between January 1984 and November 2019 at CHUP Renal Transplantation Unit. Results: In this period, 165 kidney transplants were performed, in a total of 157 patients under 18 years old. One hundred and fifty-one grafts came from cadaveric donor versus 14 from living donors. It was carried out 21 preemptive transplants, with most patients previously undergoing dialysis (50\% only peritoneal dialysis). The median dialysis waiting time for transplantation was 10 months. Ninety-two $(59 \%)$ were male patients with 13 years $(3-18)$ median age at intervention. The main etiology of kidney disease was congenital anomalies of the kidney and urinary tract (CAKUT), reported in 58\%. Donors had a median age of 17 years $(1-58)$ and terminal serum creatinine $1.00 \mathrm{mg} / \mathrm{dL}(0.30-2.10)$. The hospital stay was 14 days $(4-60)$. There were 24 episodes of tubular necrosis after transplantation, and 35 episodes of acute rejection. Renal graft and patient survival were $94 \%, 87 \%, 77 \%$ and $62 \%$ and $99 \%, 98 \%, 98 \%$ and $93 \%$ at $1,5,10$ and 20 years, respectively. Subgroups of analysis of decades has documented increasing survival rates ( $p$-value $<0.001)$. Conclusion: The results corroborate the literature and state an excellent survival associated to pediatric kidney transplantation. Although larger impact multicenter studies are needed, this analysis holistically represents the national reality of this modality.

Keywords: Kidney Transplantation; Pediatrics; Kidney Failure, Chronic; Survival Analysis. 


\section{REFERÊNCIAS}

1. Dharnidharka V, Fiorina P, Harmon W. Kidney transplantation in children. N Engl J Med 2014; 371 (6):549-58.

2. Horslen S, Barr M, Christensen L, Ettenger R, Magee J. Pediatric transplantation in the United States,1996-2005. Am J Transplant 2007;7:1339-58.

3. Branco F, Almeida F, Cavadas V, Ribeiro S, Osório L, Rocha A, et al. Pediatric kidney transplantation: a single center experience with 134 procedures. Transplantation Proceedings 2013;45:1057-9.

4. Solez K, Colvin R, Racusen L, Haas M, Sis B, Mengel $M$, et al. Banff 07 classification of renal allograft pathology: updates and future directions. Am J Transplant 2008;8:753-60.

5. Rees L, Shroff R, Hutchinson C, Fernando O, Trompeter R. Long-term outcome of pediatric renal transplantation: follow-up of 300 children from 1973 to 2000. Nephron Clin Pract. 2007;105(2):c68-76.

6. Sociedade Portuguesa de Nefrologia [homepage na Internet]. Relatório Anual do Gabinete de Registo da Sociedade Portuguesa de Nefrologia, 2018 [acesso em Outubro de 2019]. Disponível em: https://www.spnefro.pt/.
7. Sociedade Portuguesa de Pediatria [homepage na Internet]. Registo Português de IRC Pediátrica em Tratamento de Substituição Renal, 2013 [acesso em Outubro de 2019]. Disponível em: https://www.spp.pt/.

8. Kramer A, Pippias M, Noordzij M, Stel V, Andrusev A, Aparicio-Madre M, et al. The European Renal Association-European Dialysis and Transplant Association (ERA-EDTA) Registry Annual Report 2016: a summary. Clin Kidney J. 2019 Feb 26;12(5):702-20.

9. Moudgil A, Dharnidharka V, Lamb K, Meier-Kriesche $H$. Best allograft survival from Share-35 kidney donors occurs in middle-aged adults and young children - an analysis of OPTN data. Transplantation 2013;95:319-25.

10. Amaral S, Sayed B, Kutner N, Patzer R. Preemptive kidney transplantation is associated with survival benefits among pediatric patients with end-stage renal disease. Kidney Int. 2016 Nov; 90(5):1100-8.

11. Pérez-Bertólez S, Barrero R, Fijo J, Alonso V, Ojha $D$, Fernández-Hurtado $M$, et al. Outcomes of pediatric living donor kidney transplantation: A single-center experience. Pediatr Transplant. 2017 May;21(3). 